\title{
Limiting Regimes of a Three-Dimensional Buoyant Hydraulic Fracture Emerging from a Point Source
}

\author{
Andreas Möri, Lecampion Brice
}

August 3, 2021

Buoyant hydraulic fractures occur in nature as magmatic dikes and sills [1]. In industrial applications like well stimulation [2] or soil remediation [3], the emergence of buoyant fractures is undesirable and often limited by the injected volume and/or variation of in-situ stress. This class of tensile fractures is governed by a buoyancy force resulting from the density contrast between the surrounding solid and the fracturing fluid. We focus here on fluid release from a point source in an impermeable elastic media with homogeneous rock and fluid properties. The resulting buoyant force is thus constant. We combine scaling arguments and planar 3D hydraulic fracture growth simulations [4] to fully understand the emergence as well as the different propagation regimes of buoyant fractures.

For a continuous release, a family of solutions dependent on a dimensionlessviscosity $\mathcal{M}_{\hat{k}}$ exists. In the limit of large toughness $\left(\mathcal{M}_{\hat{k}} \ll 1\right)$, we retrieve a finger-like shape [5]. The stable breadth of the tail is generally akin to the PKN approximation presented in [5]. The limit of a viscosity-dominated buoyant fracture $\left(\mathcal{M}_{\hat{k}} \gg 1\right)$ has no stabilized breadth and exhibits a teardrop shape.

For the case of a finite fluid volume release, a dimensionless buoyancy $\mathcal{B}_{\bar{k}}$ controls if a buoyant fracture emerges $\left(\mathcal{B}_{\bar{k}} \geq 1\right)$ or stops and remains at depth $\left(\mathcal{B}_{\bar{k}}<1\right)$. For a finite release, a single large-time solution corresponding to the solution of [5] exists. Detailed characterization of the fracture evolution requires separation between the cases where the buoyant transition occurs during or after the release (see Fig. 1).

For natural configurations, the emerging buoyant fractures are typically viscosity-dominated, which may explain the reported discrepancy between field and laboratory measurements of rock fracture toughness. Representative values of industrial single-entry hydraulic fracturing treatments lead to buoyant fractures under homogeneous conditions, which indicate the critical importance of stress and material heterogeneities in the containment of buoyant fractures at depth. 

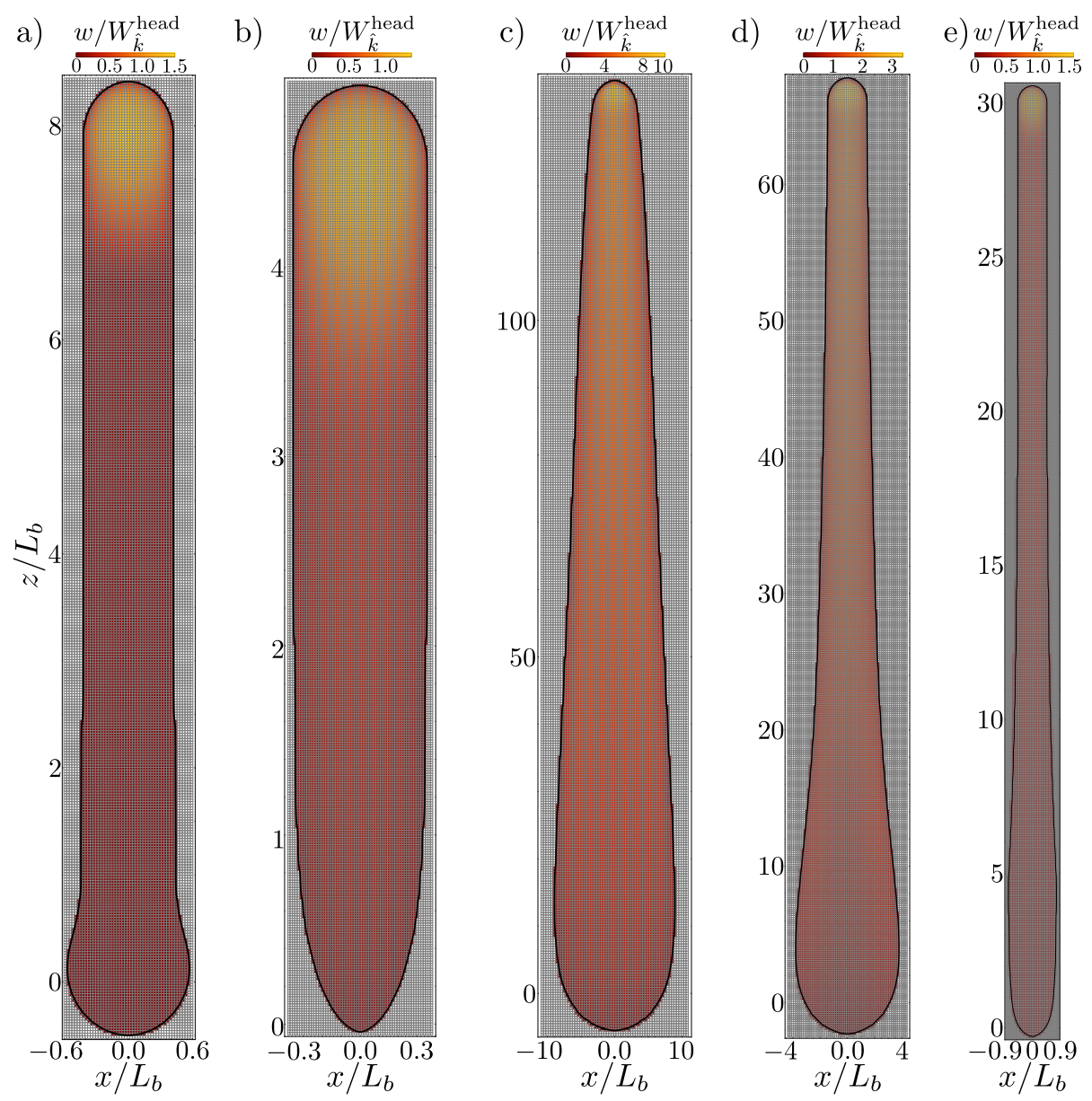

Figure 1: Different shapes of buoyant fractures emerging from a pulse release. Coordinates are scaled by the buoyancy length scale $L_{b}=K_{I c}^{2 / 3} /(\Delta \gamma)^{2 / 3}$ and opening by the characteristic head opening in the toughness-dominated limit. We give hereafter the values of $\mathcal{B}_{\bar{k}}$, and $\mathcal{M}_{\hat{k}}$ as well as the regime at the transition for the different shapes. a) $1.20,5.38 \times 10^{4}$, transition after the release, b) $1.10,7.43 \times 10^{-3}$, toughness transition during release, c) $192,5.28 \times 10^{8}$, viscous transition during release, d) $31.8,4.00 \times 10^{4}$, viscous transition during release, e) $3.35,2.24$, viscous transition during release. 


\section{References}

[1] E Rivalta, B Taisne, AP Bunger, and RF Katz. A review of mechanical models of dike propagation: Schools of thought, results and future directions. Tectonophysics, 638:1-42, 2015.

[2] M. B. Smith and C. T. Montgomery. Hydraulic Fracturing. Crc Press, 2015.

[3] Leonid N Germanovich and Lawrence C Murdoch. Injection of solids to lift coastal areas. In Proceedings of the Royal Society of London A: Mathematical, Physical and Engineering Sciences, volume 466, pages 3225-3252. The Royal Society, 2010.

[4] Haseeb Zia and Brice Lecampion. Pyfrac: A planar 3d hydraulic fracture simulator. Computer Physics Communications, page 107368, 2020.

[5] L.N. Germanovich, D. I. Garagash, Murdoch, L., and Robinowitz M. Gravity-driven hydraulic fractures. In AGU Fall meeting, 2014. 